\title{
Narrow-band GeV photons generated from an x-ray free-electron laser oscillator
}

\author{
Ryoichi Hajima, ${ }^{1, *}$ and Mamoru Fujiwara ${ }^{1,2}$ \\ ${ }^{1}$ Quantum Beam Science Center, Japan Atomic Energy Agency, Tokai, Naka, Ibaraki 3191195, Japan \\ ${ }^{2}$ Research Center for Nuclear Physics, Osaka University, Mihogaoka 10-1, Ibaraki 5670047, Japan
}

(Received 17 July 2015; published 16 February 2016)

\begin{abstract}
We propose a scheme to generate narrow-band $\mathrm{GeV}$ photons, $\gamma$-rays, via Compton scattering of hard $\mathrm{x}$-ray photons in an x-ray free-electron laser oscillator. Generated $\gamma$-rays show a narrow-band spectrum with a sharp peak, $\sim 0.1 \%$ (FWHM), due to large momentum transfer from electrons to photons. The $\gamma$-ray beam has a spectral density of $\sim 10^{2} \mathrm{ph} /(\mathrm{MeV} \mathrm{s})$ with a typical set of parameters based on a 7-GeV electron beam operated at 3-MHz repetition, Such $\gamma$-rays will be a unique probe for studying hadron physics. Features of the $\gamma$-ray source, flux, spectrum, polarization, tunability and energy resolution are discussed.
\end{abstract}

DOI: 10.1103/PhysRevAccelBeams.19.020702

\section{INTRODUCTION}

Generation of high-energy photons via collision of relativistic electrons and laser photons is known as inverse Compton scattering or laser Compton scattering (LCS). LCS photon sources have been developed and used in a wide range of photon energies from $\mathrm{keV}$ to $\mathrm{GeV}$, since LCS photons are energy tunable according to electron beam energy, laser wavelength and collision geometry [1]. Typical applications of LCS photon sources are imaging for medical application in $\mathrm{keV}$ [2], science of photonuclear reaction and its utilization for nuclear industry in $\mathrm{MeV}$ [3,4], and hadron physics in GeV [5]. Since performance of such photon sources is evaluated by average photon flux $(\mathrm{ph} / \mathrm{s})$ or average spectral density $[\mathrm{ph} /(\mathrm{eVs})]$, flux and spectral density discussed in the present paper are time averaged values.

Most of LCS photon sources in $\mathrm{keV}$ and $\mathrm{MeV}$ energies rely on both the electron beam energies from several tens $\mathrm{MeV}$ to $1 \mathrm{GeV}$ and the incident laser beams with wavelengths ranging from infrared to ultraviolet. In these LCS sources, scattering of laser photons in the electron rest frame is recognized as Thomson scattering, because a laser photon energy in the rest frame is much smaller than the electron rest mass. The Thomson-scattered photon has an almost flat spectrum up to the maximum energy determined by the electron and laser photon energies and the collision angle. A spectral bandwidth of LCS beams at an experimental area is controllable by collimators on the beam path, because the energy of scattered photon has a correlation with its scattering angle. Applications of $\mathrm{keV}$

\footnotetext{
"hajima.ryoichi@jaea.go.jp

Published by the American Physical Society under the terms of the Creative Commons Attribution 3.0 License. Further distribution of this work must maintain attribution to the author(s) and the published article's title, journal citation, and DOI.
}

and MeV LCS beams usually make use of a quasimonochromatic LCS beam with a $1 \%-10 \%$ bandwidth by selecting a specific energy with a collimator inserted into the beam path.

For a LCS beam in photon energies of $\mathrm{GeV}$, selection of photon energy by a collimator is not available due to a small divergence of LCS beam. In the case of the LCS beam line at SPring-8, 1.5-3.0 GeV $\gamma$-rays are generated with a flux of $10^{6} \mathrm{ph} / \mathrm{s}$, and the photon energies are individually determined by measuring recoiled electron energies from Compton scattering instead of photon energy selection with a collimator $[5,6]$.

In this paper, we propose a scheme to generate narrowband $\gamma$-rays via Compton scattering of hard $\mathrm{x}$-ray photons in an X-ray free-electron laser oscillator (XFELO). Due to the large momentum transfer from an electron to a scattered photon at the highly relativistic collision, the photon spectrum deviates from the Thomson spectrum and shows a high-energy peak as described by the Klein-Nishina formula. The XFELO-based Compton $\gamma$-ray source (XFELO- $\gamma$ ) produces a multi-GeV $\gamma$-ray beam with extremely narrow bandwidth, $\sim 0.1 \%$ (FWHM), and a high spectral density, $\sim 10^{2} \mathrm{ph} /(\mathrm{MeV} \mathrm{s})$. Such a $\gamma$-ray beam will be a unique probe for studying hadron physics.

\section{GEV PHOTON GENERATION FROM XFELO}

An x-ray free-electron laser oscillator (XFELO) is adopted for a $\mathrm{GeV}$ photon source in the present paper. XFELO has been proposed to obtain a hard $\mathrm{x}$-ray laser from a multi-GeV electron beam traversing an undulator, and is operated with a low-loss oscillator consisting of crystal mirrors [7]. The obtained $\mathrm{x}$-ray beam is coherent in time and space, and has characteristics with the pulse length of ps and with the repetition of $1-100 \mathrm{MHz}[7,8]$. XFELO produces hard x-ray pulses with a narrow bandwidth $\sim 2 \mathrm{meV}$, the peak spectral brightness comparable to that of self-amplified spontaneous emission (SASE) from a 
(a)
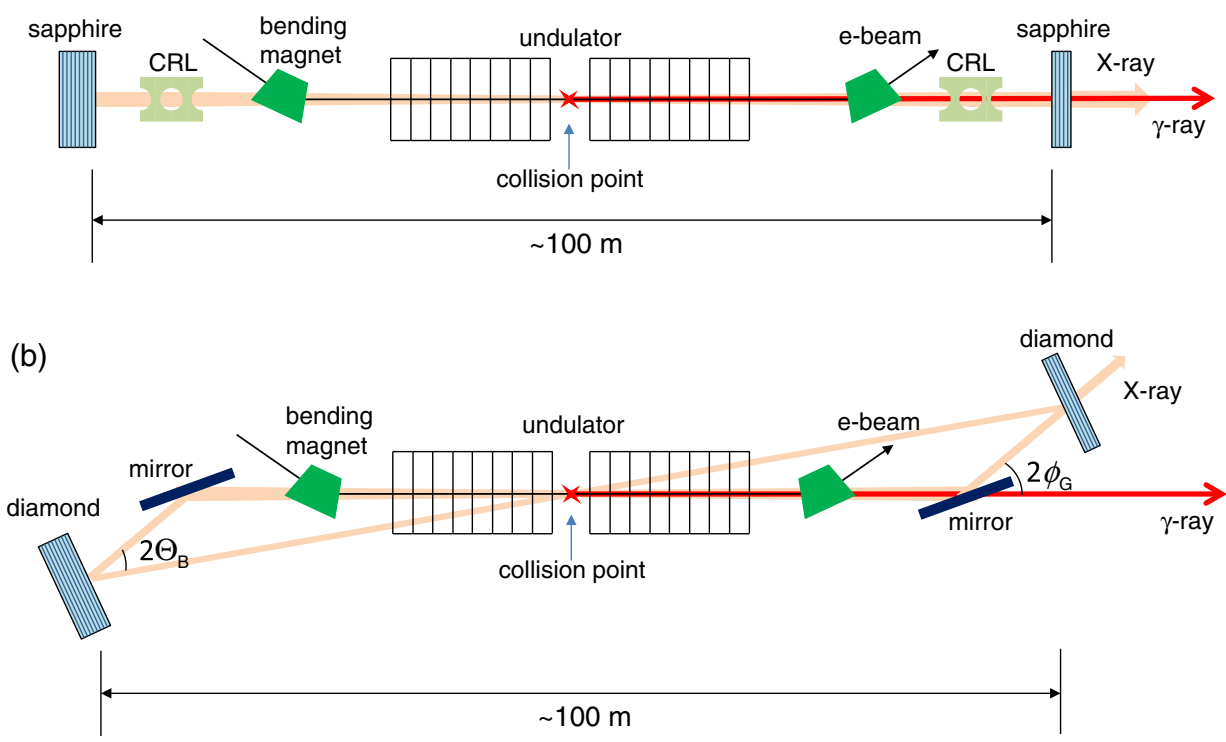

FIG. 1. Schematic views of x-ray FEL oscillators. (a) An oscillator with two normal-incidence sapphire crystals and compound refractive lenses (CRL). (b) An oscillator consisting of two diamond crystals with nonzero Bragg angle, $\Theta_{B} \neq 0$ and two focusing mirrors with a small grazing angle $\phi_{G}<1 \mathrm{mrad}$.

high-gain $\mathrm{x}$-ray FEL and the average brightness higher than SASE by several orders of magnitude $[7,8]$. Owing to this excellent performance, construction of XFELOs is proposed as a part of the energy-recovery linac X-ray light source [9] and an extension of the x-ray SASE FEL $[10,11]$. We present that XFELO is able to produce a multi-GeV narrow-band $\gamma$-ray beam with a collision of x-ray photons and relativistic electrons in the $\mathrm{x}$-ray oscillator.

We consider two types of configurations for XFELO $\gamma$ as shown in Fig. 1. A two-mirror XFEL oscillator, Fig. 1(a), is the baseline design of XFELO- $\gamma$ in the present study. The $\mathrm{x}$-ray oscillator utilizes two normal-incidence flat sapphire $\left(\alpha-\mathrm{Al}_{2} \mathrm{O}_{3}\right)$ crystals in combination with compound refractive lenses (CRL). X-ray photons are scattered by electrons at a dispersion free section between two undulator segments in order to avoid a jitter in the direction of gamma photons caused by Compton scattering from wiggling electrons. Compton scattering in the undulator magnet is discussed later.

In addition to the baseline design, we consider a fourmirror oscillator, Fig. 1(b), to study effects of noncollinear collision of electrons and x-ray photons, because such noncollinear collision is specific to XFELO $-\gamma$ and has never been used in Compton sources based on UV-FELs. The oscillator consists of two flat diamond mirrors with nonzero Bragg angle, $\Theta_{B} \neq 0$, and additional two mirrors, which are not necessarily made of diamond. In the four-mirror oscillator, an x-ray beam is traversing the undulator together with an electron beam and then reflected back along a noncollinear path. The collision of $\mathrm{x}$-ray photons and electrons can be made at the center of the undulator as shown in Fig. 1(b). Since the focusing mirrors should be set up for a grazing angle smaller than the critical angle for the total reflection, $\phi_{G}<\phi_{\mathrm{cr}} \sim 1 \mathrm{mrad}$ for $12 \mathrm{keV} \mathrm{x}$ rays, the collision angle of Compton scattering becomes smaller than $1 \mathrm{mrad}$. Several design studies of XFELO for hard $\mathrm{X}$-ray generation have been carried out, where the electron energy ranges from $3.5 \mathrm{GeV}$ [12] to $10 \mathrm{GeV}$ [7]. We have chosen a typical set of parameters listed in Table I, for the baseline design of a $\mathrm{GeV}$ photon source. The parameters are similar to a design shown in Refs. [7,8].

Generation of $\mathrm{GeV}$ photons is available in other types of XFELO with four-crystal mirrors and focusing elements designed for tuning the x-ray energy [8]. We, however, do not discuss such configurations because the $\mathrm{x}$-ray energy tunability is not essential for the present purpose.

Free-electron laser oscillators for UV and vacuum ultraviolet have been employed as LCS photon sources for $\mathrm{MeV}$ $\gamma$-rays [13-15]. The high intensity $\gamma$-ray source $(\mathrm{HI} \gamma \mathrm{S})$ at Duke University is based on Compton scattering of UV photons generated in a FEL oscillator by electrons at a $1.2-\mathrm{GeV}$ storage ring. The $\gamma$-ray source routinely provides quasimonochromatic photons at $1-100 \mathrm{MeV}$ [3]. A freeelectron laser oscillator is suitable for a LCS source, because synchronization and spatial overlap of laser photons and electrons are automatically obtained. In addition, a use of intracavity laser photons leads to high flux LCS beams.

In the XFELO- $\gamma$, the aforementioned advantages of FEL oscillator for a LCS source are totally inherited. Moreover, a narrow band $\gamma$-ray beam is obtained from the nature of Compton scattering as follows.

A schematic view of laser Compton scattering is shown in Fig. 2, where an electron with an energy of $E_{e}$ collides 
TABLE I. Parameters of the XFELO for the baseline design of XFELO- $\gamma$.

\begin{tabular}{ll}
\hline Electron beam & $7 \mathrm{GeV}$ \\
Energy $\left(E_{e}\right)$ & $40 \mathrm{pC}$ \\
Bunch charge $(Q)$ & $1.4 \mathrm{MeV}$ \\
rms energy spread $\left(\sigma_{\Delta E}\right)$ & $0.082 \mathrm{~mm}$-mrad \\
Normalized rms emittance $\left(\varepsilon_{n}\right)$ & $2 \mathrm{ps}$ \\
rms bunch length $\left(\tau_{e}\right)$ & $3 \mathrm{MHz}$ \\
Bunch repetition $(f)$ & \\
Undulator & 1.414 \\
Undulator parameter $(K)$ & $1.88 \mathrm{~cm}$ \\
Pitch $\left(\lambda_{u}\right)$ & 3000 \\
The number of periods $\left(N_{u}\right)$ & \\
FEL & $1 \AA$ \\
Wavelength $(\lambda)$ & $12.3 \mathrm{keV}$ \\
Energy $\left(E_{1}\right)$ & $100 \mathrm{~m}$ \\
Cavity length $\left(L_{c}\right)$ & $50 \%$ \\
Small signal gain & $17 \%$ \\
Round-trip loss & $4 \%$ \\
Out-couple & $0.85 \mathrm{ps}$ \\
rms pulse length $\left(\tau_{X}\right)$ & $2.3 \mathrm{meV}$ \\
rms energy spread $\left(\sigma_{\Delta E_{1}}\right)$ & \\
Collision parameters & $10 \mathrm{~m}$ \\
Beta function $\left(\beta^{*}\right)$ & $10 \mathrm{~m}$ \\
Rayleigh length $\left(Z_{R}\right)$ & $7.7 \mu \mathrm{m}$ \\
Electron beam rms size $\left(\sigma_{e}\right)$ & $0.77 \mu \mathrm{rad}$ \\
Electron beam rms divergence $\left(\sigma_{e}{ }^{\prime}\right)$ & $8.9 \mu \mathrm{m}$ \\
X-ray beam rms size $\left(\sigma_{X}\right)$ & $0.89 \mu \mathrm{rad}$ \\
X-ray beam rms divergence $\left(\sigma_{X}{ }^{\prime}\right)$ & $2.5 \times 10^{8}$ \\
The number of electrons $\left(N_{e}\right)$ & $2.0 \times 10^{10}$ \\
The number of x-ray photons $\left(N_{X}\right)$ & \\
\hline \hline & \\
\hline
\end{tabular}

with a laser photon with an energy of $E_{1}$ to produce a scattered photon with an energy of $E_{2}$. The energy of scattered photon is given as

$$
\epsilon_{2}=\epsilon_{1} \frac{1-\beta \cos \theta_{1}}{1-\beta \cos \theta_{2}+\left(\epsilon_{1} / \gamma_{e}\right)(1-\cos \theta)},
$$

where $\gamma_{e}=E_{e} / m c^{2}, \epsilon_{1}=E_{1} / m c^{2}$ and $\epsilon_{2}=E_{2} / m c^{2}$ are the electron, incident and scattered photon energies normalized by the electron rest mass, respectively, $\beta$ is the electron velocity in units of light speed, $\theta_{1}$ and $\theta_{2}$ are the angles between the direction of motion of the electrons and incident and scattered photons, $\theta$ is the angle between the incident and scattered photons.

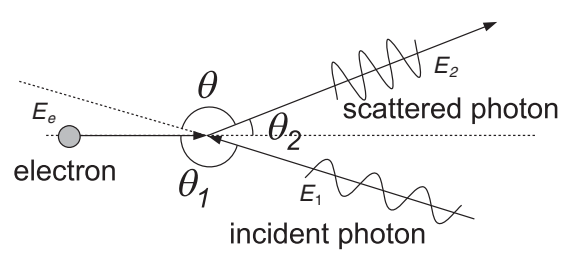

FIG. 2. A schematic view of laser Compton scattering.
For the head-on collision at $\theta_{1}=\pi$, the maximum energy of scattered photons is

$$
\epsilon_{2}^{\max }=\frac{4 \gamma_{e}^{2} \epsilon_{1}}{1+4 \gamma_{e} \epsilon_{1}}
$$

and the energy differential cross section is given as [16]

$$
\begin{aligned}
\frac{d \sigma_{c}}{d \epsilon_{2}}= & \frac{\pi r_{0}^{2}}{2} \frac{1}{\gamma_{e}^{2} \epsilon_{1}}\left[\frac{1}{4 \gamma_{e}^{2} \epsilon_{1}^{2}}\left(\frac{\epsilon_{2}}{\gamma_{e}-\epsilon_{2}}\right)^{2}-\frac{1}{\gamma_{e} \epsilon_{1}}\left(\frac{\epsilon_{2}}{\gamma_{e}-\epsilon_{2}}\right)\right. \\
& \left.+\frac{\gamma_{e}-\epsilon_{2}}{\gamma_{e}}+\frac{\gamma_{e}}{\gamma_{e}-\epsilon_{2}}\right],
\end{aligned}
$$

where $\sigma_{c}$ is the cross section of Compton scattering and $r_{0}$ is the classical electron radius.

In the case of $\gamma_{e} \epsilon_{1} \ll 1$, the collision of electron and photon is close to the Thomson scattering cross section, and the scattered photon spectrum becomes almost flat for $0<\epsilon_{2}<\epsilon_{2}^{\max }$. The scattered photon spectrum is, however, significantly different for $\gamma_{e} \epsilon_{1} \gg 1$, and has a sharp peak at $\epsilon_{2}=\epsilon_{2}^{\max }$. Figure 3 shows these spectral behaviors of Compton scattered photons for electron beam energy of $7 \mathrm{GeV}$ and incident laser photon energies of $E_{1}=1.2 \mathrm{eV}$, $120 \mathrm{eV}$ and $12 \mathrm{keV}$. For the incident laser photon energy of $12 \mathrm{keV}$, Eqs. (2) and (3) indicate that scattered photons have the maximum energy of $E_{2}=6.995 \mathrm{GeV}$ with the bandwidth of $0.075 \%$ (FWHM).

Generation of a $\gamma$-ray beam utilizing Compton scattering of $\mathrm{x}$-ray photons from undulator radiation reflected back to the electron storage ring has been proposed $[17,18]$. Realistic experimental attempts, however, have not been made due to difficulties in obtaining efficient collision of $\mathrm{X}$-ray photons and electrons at a small focus spot. On the other hand, the XFELO- $\gamma$ becomes a feasible apparatus to

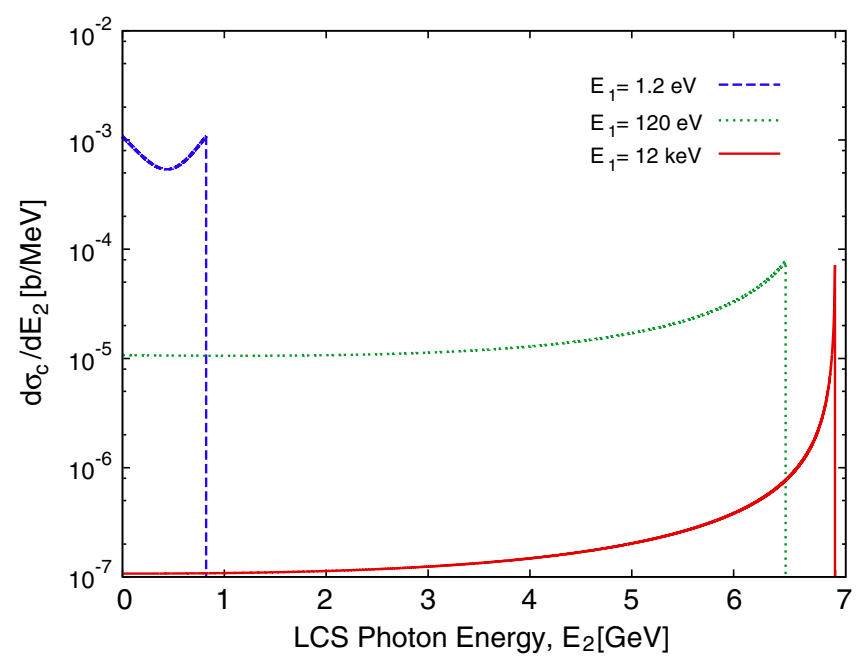

FIG. 3. Energy-differential cross section of Compton scattering for $7 \mathrm{GeV}$ electrons and laser photons at three different energies of $1.2 \mathrm{eV}, 120 \mathrm{eV}$ and $12 \mathrm{keV}$. 
achieve efficient and stable collision of $\mathrm{GeV}$ electrons and hard x-ray photons as demonstrated in MeV-photon generation at UV FEL oscillators [13-15].

\section{PERFORMANCE OF THE $\gamma$-RAY SOURCE}

\section{A. Parameters of XFELO- $\gamma$}

The performance of the XFELO- $\gamma$ has been evaluated by both analytical and numerical calculations. We assume an XFELO with sapphire mirrors shown in Fig. 1(a) with the parameters similar to Refs. [7,8] and the electron beam parameters suggested in Ref. [19]: electron energy is chosen at $E_{e}=7 \mathrm{GeV}$ to produce an x-ray beam of $1 \AA$ All the parameters of the XFELO are summarized in Table I. The X-ray beam in the cavity has a transverse mode of Gaussian distribution with the waist at the undulator center. The electron beam envelope parameter at the waist is chosen to be equal to the Rayleigh length of $\mathrm{x}$-ray beam, $\beta^{*}=Z_{R}=10 \mathrm{~m}$. The property of x-ray pulse after the lasing with assuming the cavity round-trip loss of $17 \%$ including $4 \%$ out-coupling is: $\mathrm{x}$-ray wavelength $\lambda=1 \AA$, x-ray intracavity backward photons per pulse $N_{X}=2.0 \times 10^{10}$, x-ray pulse length and energy width $\tau_{X}=0.85 \mathrm{ps}, \sigma_{\Delta E_{1}}=2.3 \mathrm{meV}$, respectively. We assume the repetition of XFELO at $3 \mathrm{MHz}$ so that an electron bunch has a collision with a backward X-ray pulse at the center of a 100-m-long cavity.

\section{B. Flux of the $\gamma$ beam}

The flux of the LCS $\gamma$-ray, $\mathcal{F}$, is calculated using the cross section of Compton scattering $\sigma_{c}$ in Eq. (3) and the luminosity $\mathcal{L}$ as $\mathcal{F}=\sigma_{c} \mathcal{L}$. The luminosity for collision of electron and laser beams at a small crossing angle, $\theta_{1}=\pi+\phi, \phi \ll 1$, is expressed by [20]

$$
\mathcal{L}=\frac{f N_{e} N_{X} \cos (\phi / 2)}{2 \pi \sqrt{\sigma_{e, y}^{2} \sigma_{L, y}^{2}} \sqrt{\left(\sigma_{e, x}^{2}+\sigma_{L, x}^{2}\right) \cos ^{2}(\phi / 2)+\left(\sigma_{e, z}^{2}+\sigma_{L, z}^{2}\right) \sin ^{2}(\phi / 2)}},
$$

where $f$ is the collision frequency, $N_{e}$ and $N_{X}$ are the number of electrons per bunch, and the number of photons in the laser pulse, $\sigma_{e, x}, \sigma_{e, y}, \sigma_{e, z}$ and $\sigma_{L, x}, \sigma_{L, y}, \sigma_{L, z}$ are the rms size of the electron and laser beam at the collision spot. We assume that the collision happens in the $x-z$ plane.

The luminosity of the XFELO- $\gamma$ of the two-mirror configuration [Fig. 1(a)] with the parameters listed in Table $\mathrm{I}$ is found to be $\mathcal{L}=1.7 \times 10^{30} \mathrm{~cm}^{-2} \mathrm{~s}^{-1}$. The cross section of Compton scattering integrated over a finite energy bandwidth (BW) of scattered $\gamma$-ray photon can be calculated from Eq. (3), and is found to be $\sigma_{c}(0.1 \% \mathrm{BW})=3.2 \times 10^{-4} \mathrm{~b}, \sigma_{c}(1 \% \mathrm{BW})=1.0 \times 10^{-3} \mathrm{~b}$ and $\sigma_{c}(100 \% \mathrm{BW})=2.9 \times 10^{-3} \mathrm{~b}$ for the bandwidth of 0.1 , 1 and $100 \%$. The $\gamma$-ray fluxes are, thus, $\mathcal{F}(0.1 \% \mathrm{BW})=$ $540 \mathrm{ph} / \mathrm{s}, \quad \mathcal{F}(1 \% \mathrm{BW})=1700 \mathrm{ph} / \mathrm{s}, \quad \mathcal{F}(100 \% \mathrm{BW})=$ $4900 \mathrm{ph} / \mathrm{s}$.

In the four-mirror XFELO- $\gamma$ [Fig. 1(b)], the luminosity is reduced due to small angle collision of $\phi \sim 1 \mathrm{mrad}$. We can evaluate the luminosity for a small angle collision using Eq. (4) and find that the luminosity reduction is negligible for a crossing angle of $\phi=1 \mathrm{mrad}$, reduction of $0.02 \%$ from the head-on collision.

\section{Bandwidth of the $\gamma$ beam}

In the generation of LCS beams for $\mathrm{keV}$ and $\mathrm{MeV}$ photon energies, quasimonochromatic photon beams are obtained with a collimator to restrict the scattering angle of photons reaching to an experimental station.

For LCS sources operated with a small collimator, energy width of a collimated LCS beam is given as

$$
\frac{\sigma_{\Delta \gamma}}{E_{\gamma}}=\sqrt{\left(\gamma_{e} \theta_{\mathrm{rms}}\right)^{4}+\left(\frac{\sigma_{\Delta \gamma}}{E_{\gamma}}\right)_{\text {inhomo. }}^{2}},
$$

where $\theta_{\text {rms }} \ll 1$ is rms acceptance of the collimator and $\left(\frac{\sigma_{\Delta \gamma}}{E_{\gamma}}\right)_{\text {inhomo }}$. is inhomogeneous spectral dilution of LCS beams due to energy spread and divergence of electron and laser beams, which is given by [21]

$$
\begin{aligned}
& \left(\frac{\sigma_{\Delta \gamma}}{E_{\gamma}}\right)_{\text {inhomo. }} \\
& =\sqrt{4\left(\frac{\sigma_{\Delta E}}{E_{e}}\right)^{2}+\left(\frac{\varepsilon_{n}}{\sigma_{e}}\right)^{4}+\left(\frac{\sigma_{\Delta E_{1}}}{E_{1}}\right)^{2}+\left(\frac{\lambda}{4 \pi \sigma_{X}}\right)^{4}},
\end{aligned}
$$

where $\sigma_{\Delta E}$ and $\varepsilon_{n}$ are electron energy spread and normalized emittance, respectively, $\sigma_{\Delta E_{1}}, \lambda$, are energy spread and wavelength of the incident laser photons, respectively. Detailed studies of Compton scattering in the sixdimensional phase space have been conducted analytically and numerically [22-24], which show an electron beam with small emittance and small energy spread is essential for obtaining narrow-band LCS beams with $\mathrm{keV}$ and $\mathrm{MeV}$ energies.

On the other hand, generation of narrow-band $\gamma$-rays from the XFELO- $\gamma$ is attributed to the nature of Compton scattering, not to the collimation with a small aperture. In the following, we see dilution of the $\gamma$-ray spectrum due to finite energy spread and divergence of both electron and $\mathrm{x}$-ray beams in the XFELO $\gamma$. 
We consider nearly head-on collision of electron and $\mathrm{x}$-ray beams at an angle, $\theta_{1}=\pi+\phi, \phi \ll 1$. The scattered photon has the maximum energy at $\theta_{2}=0$ :

$$
\epsilon_{2}^{\max } \simeq \frac{\epsilon_{1}\left[1+\beta\left(1-\phi^{2} / 2\right)\right]}{1-\beta+\left(\epsilon_{1} / \gamma_{e}\right)\left(2-\phi^{2} / 2\right)} \simeq \frac{\gamma_{e}^{2} \epsilon_{1}\left(4-\phi^{2}\right)}{1+\gamma_{e} \epsilon_{1}\left(4-\phi^{2}\right)} .
$$

The shift of the maximum energy due to a nonzero collision angle of $\phi$ is given by

$$
\Delta \epsilon_{2}^{\max } \simeq-\frac{\phi^{2}}{16 \epsilon_{1}} \quad \text { for } \gamma_{e} \epsilon_{1} \gg 1 .
$$

For the 7-GeV XFELO- $\gamma$ with the parameters shown in Table I, the divergence of the electron and laser beams at the collision point is 0.77 and $0.89 \mu \mathrm{rad}$, respectively. The dilution of the $\gamma$-ray energy spectrum caused by these divergences is as small as a few $\mu \mathrm{eV}$.

Next, we evaluate the dilution of the $\gamma$-ray energy spectrum due to the energy spread of electron and laser beams. For a head-on collision of electron and laser beams, the derivative of $\epsilon_{2}^{\max }$ in Eq. (2) with respect to the laser photon energy is calculated as

$$
\frac{d \epsilon_{2}^{\max }}{d \epsilon_{1}}=\frac{4 \gamma_{e}^{2}}{\left(1+4 \gamma_{e} \epsilon_{1}\right)^{2}} .
$$

It is found that an x-ray photon energy spread of $10 \mathrm{meV}$ introduces an energy spread of $4.5 \mathrm{eV}$ in the $7-\mathrm{GeV}$ photons.

The deviation of electron energy directly alters the energy of $\gamma$-ray photons according to the following relation:

$$
\frac{d \epsilon_{2}^{\max }}{d \gamma_{e}} \approx 1 \text { for } \gamma_{e} \epsilon_{1} \gg 1
$$

In addition to the inhomogeneous spectral dilution, nonlinear spectral effects occur in Compton scattering, when the laser normalized potential $A=\lambda e E_{0} / 2 \pi m c^{2}$ approaches unity [25,26], where $\lambda$ and $E_{0}$ are the laser wavelength and the peak value of the electric field at the collision. Such nonlinear effects do not occur in the XFELO- $\gamma$, where $A \simeq 10^{-7}$ for the design parameters.

As a result, we have found that the spectrum of XFELO $-\gamma$ is only sensitive to the energy spread of electron beam, but insensitive to other parameters: divergence of the electron beam, energy spread and divergence of the laser beam.

The electron beam energy spread at the XFELO- $\gamma$ should be evaluated by taking into account the FEL interaction. For an undulator with a period of $N_{u}$, the energy spread induced by the FEL interaction is $\left(\sigma_{E} / E_{e}\right)_{\mathrm{FEL}} \sim$ $12 /\left(4 \pi N_{u}\right)$ at the end of the undulator [27]. We assume an energy spread as half of the aforementioned value at the center of undulator. For the XFELO $-\gamma$ with the parameter listed in Table I, the electron beam at the center of undulator has an energy spread of $\sigma_{E}=1.8 \mathrm{MeV}$ including the contribution of FEL interaction.

Transverse emittance also causes a spread of longitudinal velocities in an undulator as a result of the fact that each electron has a different trajectory of wiggling motion according to its initial transverse position and momentum at the undulator entrance. It is known that this longitudinal velocity spread is one of the sources of FEL gain reduction. For the generation of $\gamma$-ray beam with Compton scattering in an FEL oscillator, transverse emittance introduces a finite spot size and a finite divergence of electrons at the collision point, but does not affect the electron energy that determines energies of scattered $\gamma$-ray photons.

\section{Numerical simulations}

A numerical evaluation of the XFELO- $\gamma$ performance was conducted with a simulation code CAIN [28]. In the simulations, we can evaluate the $\gamma$-ray properties including the effects of energy spread of electrons and finite divergence of electron and x-ray beams at the collision. Note that the CAIN simulations do not include the energy spread of x-ray photons, but we have seen that this effect is negligibly small.

Figure 4 shows the $\gamma$-ray spectrum obtained from the numerical simulation together with analytically calculated spectra for the two-mirror XFELO- $\gamma$ of the baseline design. Two curves of the analytical results correspond to a spectrum calculated using Eq. (3) and a spectrum convoluted by electron energy spread, $\sigma_{E}=1.8 \mathrm{MeV}$, respectively. The numerical result is in good agreement with the analytical one convoluted with the electron energy spread. This agreement also supports the fact that the $\gamma$-ray



FIG. 4. $\gamma$-ray spectra of the two-mirror 7-GeV XFELO- $\gamma$ calculated by an analytical formula without electron energy spread (green) and with energy spread (red), and numerical simulation by CAIN (blue). 


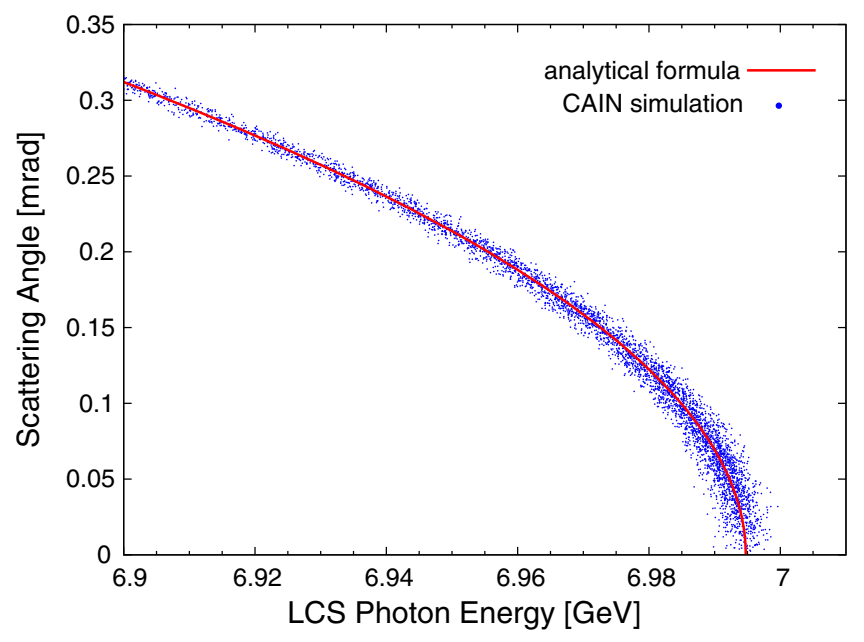

FIG. 5. Correlation of $\gamma$-ray energy and scattering angle in the two-mirror 7-GeV XFELO- $\gamma$ calculated by an analytical formula without electron energy spread (red line) and numerical simulation by CAIN (blue dots).

bandwidth is insensitive to divergence of electrons and laser photons at the collision.

Figure 5 shows the correlation of $\gamma$-ray energy and scattering angle in the XFELO $\gamma$ calculated from the analytical formula, Eq. (1) and the CAIN simulation. We can see that the scattering angle is correlated with the $\gamma$-ray photon energy, but the correlation differs from that of usual LCS photon sources at $\mathrm{keV}$ and $\mathrm{MeV}$ energies, in which photon energy becomes half of the maximum at the scattering angle of $1 / \gamma_{e}$.

The numerically calculated performance of the XFELO $-\gamma$ of baseline design is summarized in Table II.

In order to confirm the effects of noncollinear Compton scattering, the $\gamma$-ray flux and spectrum of the four-mirror XFELO- $\gamma$ was also calculated by CAIN. In the simulation, we assume the collision parameters, the number of electrons and $\mathrm{x}$-ray photons, the size and divergence of electron and $\mathrm{X}$-ray beams, same as the two-mirror XFELO- $\gamma$, but the small crossing angle at the collision, $\phi=1 \mathrm{mrad}$. We found that the flux and spectrum of the four-mirror XFELO $\gamma$ with a small crossing angle at the collision, $\phi=1 \mathrm{mrad}$ are identical to those of the two-mirror XFELO- $\gamma$ as shown in Fig. 6, because the small crossing angle does not affect the luminosity. In a practical design of a four-mirror XFELO- $\gamma$, luminosity could be improved by

TABLE II. Calculated performance of the XFELO- $\gamma$ with parameters listed in Table I.

\begin{tabular}{ll}
\hline \hline Repetition & $3 \mathrm{MHz}$ \\
Peak energy & $6.9922 \mathrm{GeV}$ \\
Bandwidth (FWHM) & $12 \mathrm{MeV}$ \\
Flux (100\% BW) & $4900 \mathrm{ph} / \mathrm{s}$ \\
Flux (1\% BW) & $1700 \mathrm{ph} / \mathrm{s}$ \\
Flux (0.1\% BW) & $460 \mathrm{ph} / \mathrm{s}$ \\
\hline \hline
\end{tabular}

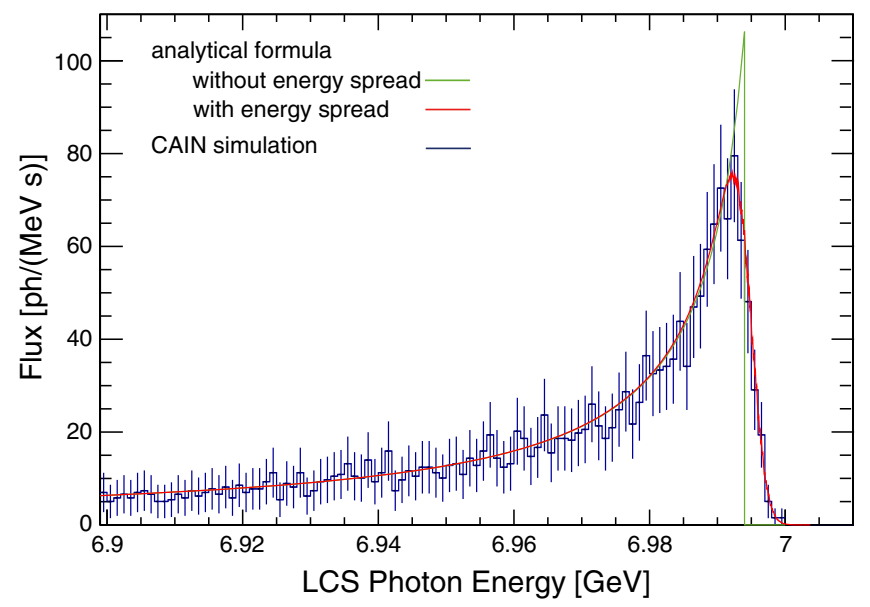

FIG. 6. $\gamma$-ray spectra of the four-mirror 7-GeV XFELO- $\gamma$ calculated by an analytical formula without electron energy spread (green) and with energy spread (red), and numerical simulation by CAIN (blue).

optimizing the collision parameters, since the collision spot size of the $\mathrm{x}$-ray backward beam is not necessarily equal to the size of the forward beam at the undulator center in a four-mirror XFELO.

\section{DISCUSSION}

As seen in Figs. 3, 4 and 6, a $\gamma$-ray spectrum of XFELO- $\gamma$ has a sharp peak at the maximum energy and a low-energy tail. For $\gamma$-ray application experiments, it is possible to determine $\gamma$ photon energy by measuring the energy of recoil electron with a tagging counter, which may be accompanied by a beam-line collimator to eliminate the low-energy $\gamma$ photons at the experimental area. The energy measurement of recoiled electrons in an XFELO $\gamma$ can be conducted with chicane magnets or a small bending magnet before the main magnet to deflect the $7-\mathrm{GeV}$ electrons. Since most of the $\gamma$-ray photons are concentrated around the peak energy, we only need to measure low-energy electrons, below $100 \mathrm{MeV}$ for example. The accuracy of photon energy measurement is restricted by electron beam energy spread at the collision, 1.8 MeV (rms) for the XFELO- $\gamma$ in this paper, which is much better than the tagging system at SPring-8 with a resolution of $12 \mathrm{MeV}$ (rms) [6].

Generation of a polarized photon beam is one of the excellent features of LCS. Polarization of photons and electrons in Compton scattering can be dealt with the matrix transformation of Stokes parameters [29]. For the case of the linearly polarized incident laser, Compton scattered photons have the degree of polarization [29]:

$$
p=\frac{2}{\left(k_{1} / k_{2}\right)+\left(k_{2} / k_{1}\right)},
$$

where polarization $p$ is defined as the absolute value of the Stokes vector, $k_{1}$ and $k_{2}$ are energies of incident and 
scattered photons normalized by $m c^{2}$, respectively. Note that these photon energies must be evaluated at the electron rest frame for Compton scattering by relativistic electrons.

In the case of $\gamma_{e} \epsilon_{1} \ll 1$, the polarization of the incident photon is preserved in the scattering process, because $k_{1} / k_{2} \sim 1$ at the electron rest frame. As a result, linearly or circularly polarized photon beams can be generated from LCS with a polarized laser.

On the other hand, a scattered photon does not preserve polarization of an incident photon in the XFELO- $\gamma$ due to a large energy exchange between the incident photon and the electron. Thus, linearly polarized $\gamma$-ray beams are not available even with linearly polarized $\mathrm{x}$ rays in XFELO $\gamma$.

The circularly polarized $\gamma$-ray from XFELO- $\gamma$ is, however, available with a longitudinally polarized (or spin polarized) electron beam. In laser Compton scattering by polarized electron beams, the scattered photons are in general elliptically polarized, and circularly polarized for the backscattering $(\theta=\pi)$ from head-on collision. The degree of polarization for backscattered photons is given by [29]

$$
p=\frac{2 k_{1}\left(1+k_{1}\right)}{\left(1+2 k_{1}+2 k_{1}^{2}\right)}\left|\overrightarrow{n_{0}} \cdot \vec{S}\right|
$$

where $\vec{n}_{0}$ is the unit vector for scattered photon momentum, $\vec{S}$ is the initial spin direction of the electron. As seen in Eq. (12), the degree of polarization approaches $\left|\vec{n}_{0} \cdot \vec{S}\right|$ for high-energy incident photons, $k_{1}>1$. As a result, the scattered photons from an XFELO- $\gamma$ with a spin polarized electron beam have a high degree of polarization either left or right circular polarization, depending on whether the initial electron spin is parallel or antiparallel with the direction of the scattered photon.

The generation of the circularly polarized $\gamma$-ray from the XFELO $-\gamma$ of baseline design with a spin polarized electron beam, helicity of +1 , was confirmed by the CAIN simulation as shown in Fig. 7, in which the degree of polarization and the Stokes parameter for circular polarization of scattered photons are plotted as a function of their energy. It can be seen that photons near the high-energy peak have almost perfect circular polarization.

Next, we discuss the energy tunability and the enhancement of the $\gamma$-ray flux from the design with parameters listed in Table I. The $\gamma$-ray peak energy in an XFELO- $\gamma$ is insensitive to the $\mathrm{x}$-ray photon energy, but is determined by the electron energy. Several design studies of XFELO for hard x-ray generation have been carried out, where the electron energy ranges from $3.5 \mathrm{GeV}$ [12] to $10 \mathrm{GeV}$ [7], i.e. $\gamma$-ray peak energy can be chosen at a specific energy between 3.5 and $10 \mathrm{GeV}$. The $\gamma$-ray peak energy is further tunable continuously from the specific energy with varying the electron beam energy and undulator gap as far as FEL lasing is obtained with given undulator and crystal mirrors. According to the FEL physics, variation of the undulator
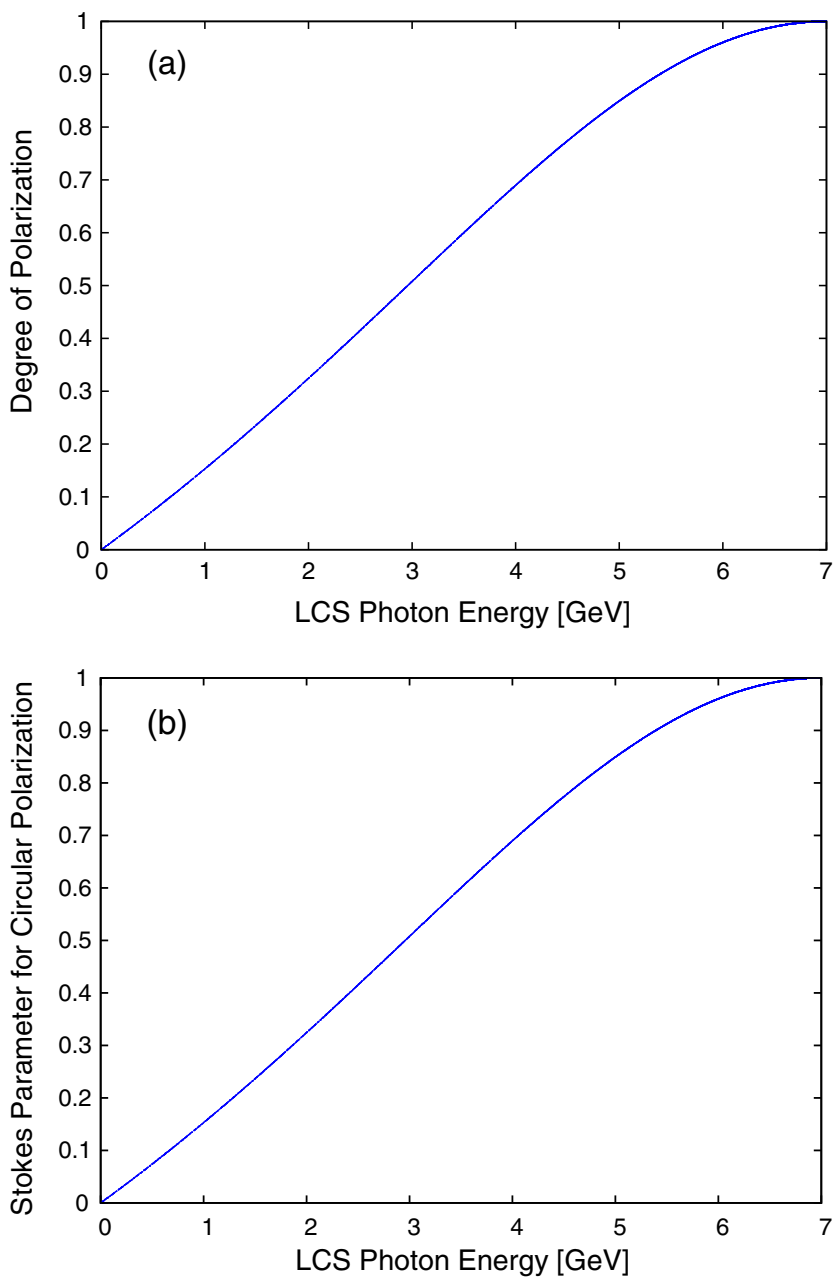

FIG. 7. Results of the CAIN simulation for $\gamma$-ray photons generated via Compton scattering with a spin polarized electron beam in the two-mirror XFELO- $\gamma$. (a) Degree of polarization, (b) The Stokes parameter for circular polarization.

parameter from $K=1.414$ in the baseline design to $K=$ 0.930 results in the variation of the electron beam energy from 7 to $5.93 \mathrm{GeV}$ to keep the FEL wavelength at $1 \AA$. The variation of the electron energy, however, introduces a FEL gain reduction of $30 \%$ from the design at $7 \mathrm{GeV}$. Available tuning range of the $\gamma$-ray peak energy by varying the electron energy depends on the parameters of the XFELO to determine FEL gain margin over the oscillator loss.

Since there is a feasibility for XFELO to be operated with the repetition of 1-100 MHz as suggested in Ref. [7], the $\gamma$-ray flux of XFELO- $\gamma$ is increased with increasing the electron beam repetition. If we allow an electron beam repetition at $90 \mathrm{MHz}$ with the same configuration as shown in Fig. 1, the $\gamma$-ray flux of XFELO- $\gamma$ with the four-mirror oscillator is enhanced by a factor of 30 compared with the $3-\mathrm{MHz}$ operation. In the two-mirror XFELO- $\gamma$, it is expected that the $\gamma$-ray flux is furthermore increased due to multiple collisions in the undulator. The enhancement factor for the two-mirror XFELO $\gamma$ is expressed to be 
$2 \eta L_{c} L_{s} / L_{b}^{2}$, where $L_{c}$ is the cavity length, $L_{b}$ is the electron bunch interval, $L_{s}$ is the length of interaction region between two bending magnets, $\eta=1+$ $\left(L_{s} / \sqrt{12} Z_{R}\right)^{2}$ is a correction factor for the variation of the collision spot size along the interaction region. The operation at $90 \mathrm{MHz}$ enables one to increase the $\gamma$-ray flux of the two-mirror XFELO- $\gamma$ by a factor of 230 compared with the 3-MHz operation. The $\gamma$-ray flux is also increased with increasing the intracavity $\mathrm{x}$-ray photons using a smaller out-coupling of the x-ray oscillator. Detailed analysis of thermal issues on crystal mirrors are necessary in a practical design of XFELO- $\gamma$, because the repetition of electron bunch and the out-coupling of the oscillator are both limited by thermal deformation of crystal mirrors. We note that the number of $\gamma$-ray photons generated from an electron bunch is much smaller than the number of electrons in a bunch even with the multiple collisions. Thus, the x-ray lasing is not affected by Compton scattering.

A $\gamma$-ray beam may have an extra divergence in an XFELO- $\gamma$ with multiple collision points along an undulator. The divergence of $\gamma$-ray photons appears according to the wiggling motion of electrons and becomes, at largest, $K / \gamma_{e}=0.10 \mathrm{mrad}$ for the $7-\mathrm{GeV}$ XFELO- $\gamma$. This divergence can be eliminated by placing collision points at an interval of integral multiple of the undulator period or placing collision points at magnet-free regions between segmented undulators, but the divergence of $\sim 0.1 \mathrm{mrad}$ seems not an issue for hadron experiments with a target of $\mathrm{cm}$ order. Degradation of the $\gamma$-ray spectrum due to the extra divergence in multiple collisions is negligibly small as described in Sec. III.

Narrow-band $\mathrm{GeV}$ photon sources similar to that presented in this study are also feasible, in principle, by utilizing single-pass SASE XFELs. A high-repetition SASE XFEL based on a cw superconducting linac is under construction to produce a high-flux X-ray beam, the number of photons per pulse of $10^{11}$ within $0.1 \%$ bandwidth, repetition of $1 \mathrm{MHz}$, photon energy of $5 \mathrm{keV}$ and electron beam energy of $4 \mathrm{GeV}$ [30]. Such x-ray and electron beams provide an opportunity of Compton scattering. For the generation of $\mathrm{GeV}$ photons from Compton scattering in SASE FELs, handling of the $\mathrm{x}$-ray beam, focusing and alignment to the collision point, is a technical challenge to overcome. Performance of a GeV photon source based on a SASE XFEL can be evaluated in the same manner as shown in this study.

\section{CONCLUSIONS}

We have proposed a narrow-band $\mathrm{GeV}$ photon source utilizing an XFEL oscillator. In the baseline design with a typical set of parameters based on a $7-\mathrm{GeV}$ electron beam operated at 3-MHz repetition, 7-GeV $\gamma$-ray photons are produced with extremely narrow bandwidth, $\sim 0.1 \%$
(FWHM), and a high spectral density, $\sim 10^{2} \mathrm{ph} /(\mathrm{MeV} \mathrm{s})$. Improvement of the flux is also possible by increasing the electron bunch repetition or by enlarging the number of $\mathrm{x}$-ray photons stored in the oscillator up to the limit of thermal deformation of the crystal mirrors. The energy of $\gamma$-ray is tunable by varying the electron beam energy as far as XFELO is lasing. A circularly polarized $\gamma$-ray beam can be generated in XFELO- $\gamma$ with a spin-polarized electron beam.

Detailed design and optimization of XFELO- $\gamma$ should be carried out in consideration of availability of smallemittance electron beams, thermal deformation of crystal mirrors, mechanical stability of a large-scale oscillator, and so on. The present design will be a starting point for these future studies.

The XFELO $-\gamma$ will open a new opportunity for studying the charmed quark (c-quark) production dynamics from the proton and the neutron which mainly consist of $u$ - and $d$-quarks. In the past, the production of $\phi, \Lambda$ and $\Sigma$ particles including strangeness quarks has been studied at the Jefferson Laboratory and at the SPring-8 [31]. In the future, new types of experiments with an XFELO- $\gamma$ will be realized to produce, i.e., the $\mathrm{J} / \Psi$ meson and charmed baryons from the $u$ - and $d$-quark medium [32].

[1] G. A. Krafft and G. Priebe, Compton sources of electromagnetic radiation, Rev. Accl. Sci. Tech. 03, 147 (2010).

[2] K. Achterhold, M. Bech, S. Schleede, G. Potdevin, R. Ruth, R. Loewen, and F. Pfeiffer, Monochromatic computed tomography with a compact laser-driven X-ray source, Sci. Rep. 3, 1313 (2013).

[3] H. R. Weller, M. W. Ahmed, H. Gao, W. Tornow, Y. K. Wu, M. Gai, and R. Miskimen, Research opportunities at the upgraded $H I \gamma S$ facility, Prog. Part. Nucl. Phys. 62, 257 (2009).

[4] R. Hajima et al., Application of Laser Compton Scattered gamma-ray beams to nondestructive detection and assay of nuclear material, Eur. Phys. J. Spec. Top. 223, 1229 (2014).

[5] M. Fujiwara, Hadron and nuclear physics with inverse Compton gamma-rays at SPring-8, Prog. Part. Nucl. Phys. 50, 487 (2003).

[6] M. Niiyama, Recent results from LEPS and prospects of LEPS II at SPring-8, Nucl. Phys. A914, 543 (2013).

[7] K.-J. Kim, Y. Shvyd'ko, and S. Reiche, A Proposal for an $\mathrm{X}$-Ray Free-Electron Laser Oscillator with an EnergyRecovery Linac, Phys. Rev. Lett. 100, 244802 (2008).

[8] K.-J. Kim and Y. Shvyd'ko, Tunable optical cavity for an X-ray free-electron-laser oscillator, Phys. Rev. ST Accel. Beams 12, 030703 (2009).

[9] R. Hajima, N. Nishimori, N. Sei, M. Shimada, and N. Nakamura, Simulations of XFELO for the KEK ERL, in Proceedings of the 34th International Free-Electron Laser Conference, Nara, Japan, 2012 (JACOW, Nara, 2012), p. 433.

[10] J. Zemella, J. Rossbach, C. P. Maag, and H. Sinn, Numerical simulations of an XFELO for the European XFEL driven by a spent beam, in Proceedings of the 
34th International Free-Electron Laser Conference, Nara, Japan, 2012 (JACOW, Nara, 2012), p. 429.

[11] T. J. Maxwell et al., Feasibility study for an X-ray FEL oscillator at the LCLS-II, in Proceedings of the 2015 Particle Accelerator Conference, Richmond, VA, 2015 (JACOW, Richmond, 2015), TUPMA028.

[12] J. Dai, H. Deng, and Z. Dai, Proposal for an X-Ray Free Electron Laser Oscillator with Intermediate Energy Electron Beam, Phys. Rev. Lett. 108, 034802 (2012).

[13] V. N. Litvinenko et al., Gamma-Ray Production in a Storage Ring Free-Electron Laser, Phys. Rev. Lett. 78, 4569 (1997).

[14] M. Hosaka, H. Hama, K. Kimura, J. Yamazaki, and T. Kinoshita, Observation of intracavity Compton backscattering of the UVSOR free electron laser, Nucl. Instrum. Methods Phys. Res., Sect. A 393, 525 (1997).

[15] M. E. Couprie, D. Nutarelli, R. Roux, B. Visentin, L. Nahon, R. Bakker, A. Delboulbé, and M. Billardon, Gamma rays produced by intra-cavity inverse Compton scattering of a storage ring free-electron laser, J. Phys. B 32, 5657 (1999).

[16] F. R. Arutyunian and V. A. Tumanian, The Compton effect on relativistic electrons and the possibility of obtaining high energy beams, Phys. Lett. 4, 176 (1963).

[17] V. Nelyubin, M. Fujiwara, T. Nakano, and B. Wojtsekhowski, Compton back-scattering of X-photons from 8-GeV electrons at SPring-8, Nucl. Instrum. Methods Phys. Res., Sect. A 425, 65 (1999).

[18] H. Ohkuma et al., Production of quasi-monochromatic $\mathrm{GeV}$ photons by Compton scattering using undulator X-ray radiation at SPring-8, in Proceedings of the 2014 Particle Accelerator Conference, Dresden, Germany, 2014 (JACOW, Dresden, 2014), p. 941.

[19] I. V. Bazarov and C. K. Sinclair, Multivariate optimization of a high brightness dc gun photoinjector, Phys. Rev. ST Accel. Beams 8, 034202 (2005).

[20] T. Suzuki, General formulae of luminosity for various types of colliding beam machines, KEK note 76-3, KEK, Tsukuba, Japan, 1976.
[21] V. Petrillo et al., Photon flux and spectrum of $\gamma$-rays Compton sources, Nucl. Instrum. Methods Phys. Res., Sect. A 693, 109 (2012).

[22] W. J. Brown and F. V. Hartemann, Three-dimensional time and frequency-domain theory of femtosecond X-ray pulse generation through Thomson scattering, Phys. Rev. ST Accel. Beams 7, 060703 (2004).

[23] C. Sun and Y. K. Wu, Theoretical and simulation studies of characteristics of a Compton light source, Phys. Rev. ST Accel. Beams 14, 044701 (2011).

[24] F. V. Hartemann, W. J. Brown, D. J. Gibson, S. G. Anderson, A. M. Tremaine, P. T. Springer, A. J. Wootton, E. P. Hartouni, and C. P. J. Barty, High-energy scaling of Compton scattering light sources, Phys. Rev. ST Accel. Beams 8, 100702 (2005).

[25] G. A. Krafft, Spectral Distributions of Thomson-Scattered Photons from High-Intensity Pulsed Lasers, Phys. Rev. Lett. 92, 204802 (2004).

[26] F. V. Hartemann, F. Albert, C. W. Siders, and C. P. J. Barty, Low-Intensity Nonlinear Spectral Effects in Compton Scattering, Phys. Rev. Lett. 105, 130801 (2010).

[27] E. L. Saldin, E. A. Schneidmiller, and M. V. Yurkov, The Physics of Free Electron Lasers (Springer-Verlag, Berlin, 1999).

[28] CAIN version 2.42; P. Chen, G. Horton-Smith, T. Ohgaki, A. W. Weidemann, and K. Yokoya, CAIN: Conglomérat d'ABEL et d'Interactions Non-linéaires, Nucl. Instrum. Methods Phys. Res., Sect. A 355, 107 (1995).

[29] W. H. McMaster, Matrix representation of polarization, Rev. Mod. Phys. 33, 8 (1961).

[30] J. N. Galayda, The Linac coherent light source-II project, in Proceedings of the 2014 Particle Accelerator Conference, Dresden, Germany, 2014 (JACOW, Dresden, 2014), p. 935.

[31] T. Nakano et al., Multi-GeV laser-electron photon project at SPring-8, Nucl. Phys. A684, 71c (2001).

[32] K. A. Olive et al. (Particle Data Group), Review of particle physics, Chin. Phys. C 38. 090001 (2014). 